\title{
A CLINICAL STUDY OF MATERNAL AND PERINATAL OUTCOME IN OLIGOHYDRAMNIOS AT DMCH, LAHERIASARAI, BIHAR
}

\begin{tabular}{l} 
Gynaecology \\
Dr. Supriya \\
Kumari \\
\hline Dr. Surya \\
Narayan* \\
\hline
\end{tabular}

\section{Dr. Kumudini Jha}

\section{Dr. Debarshi Jana}

MBBS, M.S. (Obs. \&Gynae.), Senior Resident, Department of Obstetrics and Gynaecology, Darbhanga Medical College \& Hospital, Laheriasarai, Bihar.

MBBS, M.D. (Radio-diagnosis), Senior Resident, Department of Radio-diagnosis, Darbhanga Medical College \& Hospital, Laheriasarai, Bihar. *Corresponding Author

Professor and Head of Department, Department of Obstetrics and Gynaecology, Darbhanga Medical College \& Hospital, Laheriasarai, Bihar.

Young Scientist (DST), Institute of Post-Graduate Medical Education and Research, A.J.C. Bose Road, Kolkata, West Bengal, India-700020.

\section{ABSTRACT}

Background: Oligohydramnios is one of the major causes of maternal and perinatal morbidity and mortality. Our study was aimed to study the maternal and perinatal outcome in oligohydramnios and measures needed to reduce the incidence.

Aims: To find out the incidence of oligohydramnios by ultrasonographic evaluation of AFI, obstetric risk factors associated with oligohydramnios and maternal outcome in the form of mode of delivery. The purpose of this study is also to assess congenital anomalies in fetus along with their apgar score at birth, NICU admission rates and still birth rates.

Materials And Methods: After taking consent from the women, risk factors at the time of admission were recorded. Detailed clinical history including obstetric, menstrual, past and personal history were taken.AFI to be measured using Phelan's four quadrant ultrasound technique. All the information was entered in the proforma and analyzed and observations were made and accordingly discussion and recommendations were made.

Results: The incidence of oligohydramnios was 3\%. In the present study $78.5 \%$ cases had associated obstetrical complications; acting singly or in combination for causing oligohydramnios. The incidence of congenital anomalies is $9 \%$. The LSCS was done in $47 \%$ in present study. $17.5 \%$ babies had low Apgar score (less than 7 at $5 \mathrm{~min}$ ). In our study $36 \%$ of neonates were admitted in nursery. The perinatal mortality was $15 \%$ in present study. Conclusion: Hence it can be concluded that maternal evaluation of risk factors, AFI, regular antenatal and intranatal monitoring of fetus should be done to improve maternal and neonatal outcome in oligohydramnios.

\section{KEYWORDS}

Maternal, Fetal, Oligohydramnios, AFI, Apgar

\section{INTRODUCTION}

The aquatic environment of the fetus has long remained enigma to the patient and their obstetricians. Early in developmental life the fetus becomes enclosed by the amnion and is surrounded by amniotic fluid which initially is very similar to extracellular fluid. The importance of amniotic fluid volume as an indicator of fetal status has made the amniotic fluid volume assessment an important part of antenatal fetal surveillance.

Oligohydramnios is a severe and common complication of pregnancy and the incidence of this is reported to be around 1 to $5 \%$ of total pregnancies. The accurate diagnosis of oligohydramnios has become possible by ultrasonographic examination during pregnancy. The findings of oligohydramnios can be associated with congenital fetal abnormalities,premature rupture of membranes, uteroplacental insufficiency, growth retardation, post datism, chronic abruptio placentae. Maternal illness i.e. hypertension, preeclampsia, abnormalities of twinning, history of drug intake etc.

The sequel from long standing oligohydramnios includes pulmonary hypoplasia, potter's syndrome, club foot, club hand and dislocation of hip. It is found to be associated with high incidence of maternal and perinatal morbidity and mortality. During labour the predominant mechanical function of amniotic fluid is to provide an aquatic cushion for umbilical cord. Without this cushion, compression of the cord between the fetus and the uterine wall may occur during contractions or fetal movement, this cord compression causes severe FHR decelerations which are associated with low APGAR scores and acidosis at birth, meconium staining, caesarean section and operative vaginal delivery for fetal distress.

Thus it appears prudent to evaluate amniotic fluid volume (AFV) during any assessment of fetal status whether as a part of antenatal testing protocol or in the labour suite. Successful management of this requires thorough evaluation of the gravid for hypertension, $\mathrm{Rh}$ factor, diabetes, abruption, drug history or any other significant illness. In addition, a thorough anatomic survey focusing on genitourinary tract, fetal swallowing studies and an attempt to visualise free amniotic bands should be performed and fetal karyotyping should also be considered.
This study will review the etiological factors of oligohydramnios in respect to both mother and fetus. The incidence of congenital anomalies, doppler changes and perinatal morbidity and mortality and accordingly planning antepartum and intrapartum management. Since oligohydramnios has got significant impact on neonatal outcome and maternal morbidity, it prompted us to study this condition.

\section{MATERIALAND METHODS}

The present study was conducted in the Department of Obstetrics \&Gynaecology, Darbhanga Medical College and Hospital, Laheriasarai (Bihar) during the period of June 2019 to May 2020 (1 year). The study comprised of 200 cases of oligohydramnios (AFI up to $5 \mathrm{~cm}$ ) in third trimester ( $>28$ weeks of gestation) and who presented in the Department of Obstetrics \& Gynaecology of our hospital.

\section{Inclusion Criteria}

- Sonographically proven cases of oligohydramnios, AFI up to 5 $\mathrm{cm}$.

- Gestational age more than 28 weeks

- Singleton pregnancy

\section{Exclusion Criteria}

- $\quad \mathrm{AFI}>6 \mathrm{~cm}$

- Multiple Gestation

- Patients having major respiratory, CVS or other abdominal conditions were also excluded from the study.

\section{METHODS}

Plan of activity and time chart were formulated after taking verbal consent from the women and/or relatives. Patient's socio-demographic data were obtained. Other potential explanatory variables were obtained including maternal age, booking status, PIH and other risk factors at the time of admission were recorded. Detailed clinical history including obstetric, menstrual, past and personal history were taken. Thorough general, systemic and obstetric examination was conducted. Woman's haematological profile was done. AFI to be measured using Phelan's four quadrant ultrasound technique. The uterus is arbitrarily divided into four quadrants by the umbilicus transversely and the lineanigra vertically. The largest vertical pocket free of fetal parts and umbilical cord loops in each quadrant is 
measured and sum of these measurements will give AFI in $\mathrm{cm}$. An AFI of $5-24 \mathrm{~cm}$ is normal. AFI of $<5 \mathrm{~cm}$ is considered oligohydramnios. USG at the time of admission was recorded including fetal biometry, amniotic fluid volume and color Doppler.

Study of association of various maternal factors like prolong pregnancy, hypertensive disorders of pregnancy, PROM etc was done. Study of color doppler changes, presence of congenital anomaly if any, Improvement in AFI after treatment with parentral and oral therapy was done.

Outcome was noted in the form of mode of delivery, fetal outcome, Apgar score, fetal birth weight, maturity, admission to nursery and postnatal complications, if any

All the information was entered in the proforma and analyzed and observations were made and accordingly discussion and recommendations were made.

\section{RESULTS AND DISCUSSION}

Oligohydramnios (AFI up to 5) was detected in 386 patients, so incidence of oligohydramnios found was $3 \%$ of total admission in one year.

\section{Table 1 : Incidence Of Oligohydramnios At Our Hospital}

\begin{tabular}{|l|l|l|}
\hline Total Admission & Total Deliveries & Cases of oligohydramnios \\
\hline 12854 & 10362 & 386 \\
\hline
\end{tabular}
12854

10362

386

Table 2 :maternal Factors Associated With Oligohydramnios

\begin{tabular}{|l|l|l|}
\hline Maternal Factors & Total No. & \% n=200 \\
\hline Post term pregnancy $(>42$ weeks $)$ & 1 & 0.5 \\
Prolonged pregnancy $(>40-42$ weeks) & 33 & 16.5 \\
\hline PIH & 42 & 21 \\
Gestational Hypertension & 26 & 13 \\
Preeclampsia & 15 & 7.5 \\
Eclampsia & 1 & 0.5 \\
\hline PROM & 17 & 8.5 \\
\hline Malnutrition + Anaemia & 110 & 55 \\
\hline Breech & 21 & 10.5 \\
\hline Chorioamnionitis & 1 & 0.5 \\
\hline Chronic renal disease & 0 & 0 \\
\hline Chronic abruptio & 0 & 0 \\
\hline
\end{tabular}

The different obstetric risk factors associated with oligohydramnios were found to be $34(17 \%)$ cases of postdated pregnancy, out of which 1 of post term (more than $42 \mathrm{wks}$ ) and 33 of prolonged pregnancy, 42 (21\%)cases of PIH, out of which $26(13 \%)$ cases were of gestational hypertension, $15(7.5 \%)$ were of preeclampsia, $1(0.5 \%)$ cases wereof eclampsia, $17(8.5 \%)$ cases were of PROM, $110(55 \%)$ cases were associated with malnutrition and anaemia,21(10.5\%)cases were ofbreech presentation, $1(0.5 \%)$ case was of chorioamnionitis.

Incidence of congenital anomalies was $9 \%$ i.e. 18 cases with congenital anomalies out of 200 total cases. Out of these urinary tract system was most common(14), potter syndrome (1),amniotic band syndrome(1), CTEV(1), hydrocephalus(1)

There was high incidence of cesarean section 94 cases (47\%). There was high incidence of low Apgar score at birth both at $1 \mathrm{~min}(35 \%)$ and $5 \min (17.5 \%)$. There is high rate of admission of babies in nursery 72 babies (36\%). There is high incidence of still birth (5\%) out of which (FSB 3\% and MSB 2\%).

Table 3 : Associated Of Oligohydramnios With Congenital Anomalies

\begin{tabular}{|l|l|l|}
\hline Name of Congenital anomaly & No. of cases $(\mathbf{n}=\mathbf{2 0 0})$ & Percentage \\
\hline Urinary Tract & 14 & 7 \\
\hline Hydronephrosis/hydroureter & 9 & 4.5 \\
\hline Renal agenesis & 2 & 1 \\
\hline Renal ectasia & 1 & 0.5 \\
\hline Polycystic kidney disease & 1 & 0.5 \\
\hline Posterior urethral valve & 1 & 0.5 \\
\hline Potter syndrome & 1 & 0.5 \\
\hline CTEV & 1 & 0.5 \\
\hline Amniotic band syndrome & 1 & 0.5 \\
\hline Pulmonary hypoplasia & 2 & 1 \\
\hline Hydrocephalus & 1 & 0.5 \\
\hline
\end{tabular}

\section{GIT}

Cardiac anomaly 0
0 \begin{tabular}{l|l}
0 \\
0
\end{tabular}

Table 4 : Distribution Of Cases According To Mode Of Delivery

\begin{tabular}{|l|l|l|}
\hline Mode of delivery & No. of cases (n=200) & Percentage \\
\hline LSCS & 94 & $47 \%$ \\
\hline Vaginal delivery & 106 & $53 \%$ \\
\hline
\end{tabular}

Table 5 : Apgar Score

\begin{tabular}{|l|l|l|l|}
\hline \multirow{2}{*}{ Studies } & \multicolumn{2}{|l|}{$\begin{array}{l}\text { Occurrence of Apgar Score }<7 \text { in } \\
\text { Percentage }\end{array}$} & $\begin{array}{l}\text { Admission to } \\
\text { NICU in } \\
\text { Percentage }\end{array}$ \\
\cline { 2 - 4 } $\mathbf{1 ~ m i n .}$ & $\mathbf{5}$ min. & $7 \%$ \\
\hline Casey et al & - & - & $88.88 \%$ \\
\hline Sriya R et al & $38.88 \%$ & $9.72 \%$ & $7 \%$ \\
\hline Umber A et al & $8 \%$ & $6 \%$ & - \\
\hline Guin et al & $39 \%$ & - & $36 \%$ \\
\hline Present Study & $35 \%$ & $17.5 \%$ & \\
\hline
\end{tabular}

Table 6 : Distribution Of Cases According To Perinatal Outcome

\begin{tabular}{|l|l|l|}
\hline Perinatal Outcome & Cases $(\mathbf{n}=\mathbf{2 0 0})$ & \\
\hline Nursery admission & 72 & 36 \\
\hline Alive and healthy & 118 & 59 \\
\hline Still birth & 10 & 5 \\
\hline FSB & 6 & 3 \\
\hline MSB & 4 & 2 \\
\hline
\end{tabular}

With the easier availability of ultrasonography nowadays more cases of oligohydramnios are being identified. This helps us to be more cautious and anticipate problems especially during labour. The various outcome results are comparable to results of similar studies done both in India and abroad. Amniotic fluid volume is known to be reduced with advancing gestational age after 40 week. Perinatal morbidity and mortality are significantly increased when oligohydramnios is present at delivery. Fetal conditions that are associated with oligohydramnios are post maturity, IUGR, major fetal anomalies most commonly involving genitourinary tract.

\section{Incidence}

Study was conducted for a period of 1 year during which 12854 obstetric cases were admitted at our institution. Of them, 10362 patients delivered. Oligohydramnios (AFI <5) was detected in 386 patients as shown in table I.

So in the present study, the incidence of oligohydramnios was 3\%. Similar study by Jun Zhang et al. reported the incidence as 1.5 Casey B et al. studied 6423 patients, who underwent ultrasonography at more than 34 weeks gestation and found that $147(2.3 \%)$ cases were complicated by oligohydramnios.

\section{Maternal Risk Factors}

Obstetrical complications frequently associated with oligohydramnios werepregnancy induced hypertension $(\mathrm{PIH})$, post-datism, intrauterine growth restriction, fetal renal anomalies, prematurity and intrauterine death of the fetus. In the present study $78.5 \%$ cases had associated obstetrical complications; acting singly or in combination for causing oligohydramniosNot every patient had associated factors along with oligohydramnios. But most commonly factors which were associated were enlisted above in table II.

Hypertensive cases and post-term pregnancy were maximally associated with oligohydramnios in our study. In present study association of hypertensive disorders is $21 \%$ which is comparable to study by Sriya et al. in which its incidence was $31 \%$.

Seventeen percent cases had post-dated pregnancies in the present study. Marks and Divon (1992) has reported oligohydramnios in $11.5 \%$ of 551 pregnancies at 41 wks or greater.

\section{Congenital Anomalies}

Table III shows congenital anomalies associated with oligohy dramnios. In our study the incidence of congenital anomalies is $9 \%$ which is comparable to study done by Shetty et al with a incidence of $5.8 \%$, and study done by Golan et al with a incidence of $11 \%$ and a study of Guin et al with a incidence of $8.5 \%$.

\section{Mode Of Delivery}

Various studies show different rates of LSCS in pregnant women with amniotic fluid index of $<5 \mathrm{~cm}$. The LSCS was done in $47 \%$ in present study as shown in table IV which is compared with the situations in 
other studies. Study by Casey B et al.found that, there was increased rate of induction of labour $(42 \%)$ and Caesarean section $(32 \%)$ in oligohydramnios cases. Golan A et al. found that, the caesarean section was performed in $35.2 \%$ of pregnancies. These are comparable to my study

\section{Perinatal Outcome}

Table V shows comparison of neonates in different studies according to low Apgar score and admission to NICU.

In the present study, the Apgar score was noted at 1 and 5 minutes after birth. 17.5\% babies had low Apgar score (less than 7 at $5 \mathrm{~min}$ ) as shown in table V. Sriya R et al in their study have reported $38.8 \%$ incidence of Apgar score less than 7 at 1 minute. In a similar study by Casey B et al $(6 \%)$ babies had Apgar score of less than 3 at 5 minute. Out of these nine babies, seven died during neonatal period. Jun Zhang et al. found that an Apgar score of $<7$ at 1 minute was present in fifteen. Six babies had Apgar score of $<7$ at 5 minute. Desai P et al. found that three babies with Apgar score less than 7 at 5 minute as against only one in control group. In a similar study by Locatelli A et al. of 341 patients with oligohydramnios, found no significant difference for Apgar score of less than 7 at 5 minute in study and control group.

In our study neonates of $36 \%$ cases were admitted in nursery as shown in table VI. Sriya R et al has reported a very high incidence of NICU admission. In their study $88.88 \%$ newborns were admitted in NICU in patients having AFI $\angle 5 \mathrm{~cm}$. Casey BM et alhave reported $7 \%$ admission to the NICU in patients with $\mathrm{AFI}<5 \mathrm{~cm}$. Zhang J et al in their study have reported $29.4 \%$ admission to NICU in patients with $\mathrm{AFI}<5 \mathrm{~cm}$.

In the present study, there were 95\% live births and 5\% still births as shown in table VI. Ten percent babies died in neonatal period. The perinatal mortality was $15 \%$ in present study. Wolff $\mathrm{F}$ et al.found that the perinatal mortality in their study was 7.2\%. LiatApel-Sarid et al. found that the perinatal mortality was $9.9 \%$. The lack of amniotic fluid allows compression of fetal abdomen, which limits the movement of the diaphragm. Overall, the perinatal mortality is markedly increased in patients with oligohydramnios.

\section{CONCLUSION}

In presence of oligohydramnios thorough evaluation of the gravida for hypertension, pre-eclampsia, diabetes, chronic abruption, premature rupture of membrane, drug intake etc should be done. An amniotic fluid index of $\leq 5 \mathrm{~cm}$ detected after 28 completed weeks was associated with adverse pregnancy out come as well as indicator of poor perinatal outcome. In presence of oligohydramnios, meconium stained liquor, development of fetal distress, the rate of LSCS, low Apgar score, low birth weight, perinatal morbidity and mortality are more. There was higher incidence of congenital anomalies associated with oligohydramnios.

Determination of AFI should be used as an adjunct to other fetal surveillance methods. It helps to identify those infants at risk of poor perinatal outcome. Determination of AFI is a valuable screening test for predicting fetal distress in labour requiring caesarean section. Thorough fetal anatomic survey should be done so that the patient may be counselled for early termination and unnecessary caesarean section can be avoided for IUGR, breech, cord prolapsed. Colour Doppler study should be done in cases with AFI up to $5 \mathrm{~cm}$ so that hypoxic changes if present which are commonly associated with oligohydramnios can be detected early and accordingly early management can be done.

Regular antenatal and intranatal monitoring should be done to diagnose any fetal compromise at the earliest. Termination of pregnancy according to the balance of risk of intrauterine asphyxia against those of prematurity should be done to obtain the best outcome. One should strongly consider delivery if the fetus is likely to be mature. Caesarean section is more liberally indicated specially if there are associated adverse factors as the fetus does not tolerate the reduced oxygen supply and birth trauma encountered during vaginal delivery.

\section{REFERENCES}

1. Anna Locatelli, PatriziaVergani, Laura Toso,Maria Verderio, John C, PezzulloAlessandroGhidini. Perinatal outcome associated witholigohydramnios in uncomplicated termpregnancies. Archives of Gynaecology andObstetrics 2004;269(2)130-33.

2. Apel-Sarid L, Levy A, Holcberg G, Sheiner E. Placental pathologies associated with intrauterine fetal growth restriction complicated with and without oligohydramnios. intrauterine fetal growth restriction co
Arch gynecolObstet 2009;280:549-52.
3. Casey Brian M, McIntire DD, Bloom SL, LucasMJ, Santos R, Twickler DM et al. Pregnancy outcomes after antepartum diagnosis of oligohydramnios at or beyond 34 weeks' gestation. Am J ObstetGynecol 2000;182(4): 909-12

4. Desai Pankaj, Patel Purvi, Gupta Anjali. Decreased amniotic fluid index in low risk pregnancy: any significance? J ObstetGynecolInd 2004;54(5):464-6.

5. Golan A, Lin G, Evron S, Arieli S, Niv D, David MP. Oligohydramnios: maternal complications and fetal outcome in 145 cases. GynecolObstet Invest 1994;37(2):91-5.

6. Guin Gita, Punekar S, Lele A, KhareS . A Prospective Clinical Study of Feto-Maternal Outcome in Pregnancieswith Abnormal Liquor Volume.JObstetGynaecolI $\quad n \quad d \quad i$ 2011;61(6):652-5.

7. Jun Zhang, Troendle J, Meikle S, Klebanoff MA,Rayburn WF. Isolated oligohydramnios is not associated with adverse perinatal outcome. Bjog Mar 2004:111(3):220-5

8. Marks AD, DivonMY : Longitudinal study of the amniotic fluid index in post-dates pregnancy. ObstetGynecol 1992;79(2):229-33.

9. Moore TR. Clinical assessment of amniotic fluid. Clinobstetgynaecol 1997 Jun;40(2):303-13

10. Shetty A, Shetty S, Rai SB. A study of congenital anomalies and perinatal outcome in oligohydramnios. Int J Med Sci Public Health.2013;2(4):1081-83

11. Sriya R, Singhai S. Perinatal outcome in patients with amniotic fluid index $<5 \mathrm{~cm}$. J ObstetGynaecol India 2001;51:98-100.

12. Umber A. Perinatal Outcome in Pregnancies Complicated by Isolated Oligohydramnios at Term. Annals 2009; 15:35-7.

13. Wolff F, Schaefer R. Oligohydramnios - perinatal complications and diseases in mother and child. GeburtshilfeFrauenheilkd 1994; 54(3):139-43. 